\title{
DEVELOPING A SOCIALLY-AWARE ROBOT ASSISTANT FOR DELIVERY TASKS*
}

\author{
Carlos Flores-Vázquez ${ }^{1,2}[0000-0002-5159-9469]$, Cecilio Angulo \\ Bahon $^{2[0000-0001-9589-8199]}$, Daniel Icaza ${ }^{1[0000-0002-9989-6809]}$, and \\ Juan-Carlos Cobos-Torres ${ }^{1[0000-0001-8153-8379]}$ \\ 1 Catholic University of Cuenca, Av. Américas y G. Torrres 010101, Ecuador, \\ cfloresv@ucacue.edu.ec, \\ https://www. ucacue.edu.ec/ \\ 2 Polytechnic University of Catalonia BARCELONATECH, Intelligent Data Science \\ Artificial Intelligence Research Center, Barcelona, \\ Pau Gargallo 14 08034, España
}

\begin{abstract}
This paper discusses about elements to be considered for developing a Service Robot that performs its task in a social environment. Due to the social focus of the service, not only technical considerations are demanded in order to accomplish with the task, but also the acceptance of use for the people, who interact with all of them. As our particular research topic, we establish a taxonomy to determine the framework for the development of socially-aware robot assistants for serving tasks such as deliveries. This is a general approach to be considered for any service robot being implemented in a social context. This article presents several previous cases of the implementation of service mobile robots, their analysis and the motivation of how to solve their acceptance and use by people. Therefore, under this approach it is very important not to generate false expectations about the capabilities of the robot, because as it is explained in the state of the art analysis that very high unsatisfied expectations lead to leaving the robot unused....
\end{abstract}

Keywords: Service Robotics, Social Robotics, Human - Robot Interaction, Mobile Robotics, Technology acceptance

\section{Introduction}

A Service Robot is defined according to the ISO 8373:2012 standard [1] as a robot that performs useful tasks for humans or equipment, excluding industrial automation applications. A Professional Service Robot is similarly defined as a robot used for a commercial task, usually operated by a properly trained operator. Examples are cleaning robots for public places or delivery robots in offices

\footnotetext{
* Supported by Catholic University of Cuenca - SENESCYT - Polytechnic University of Catalonia BARCELONATECH - GIRVyP Visible Radiation and Prototyping Research Group
} 


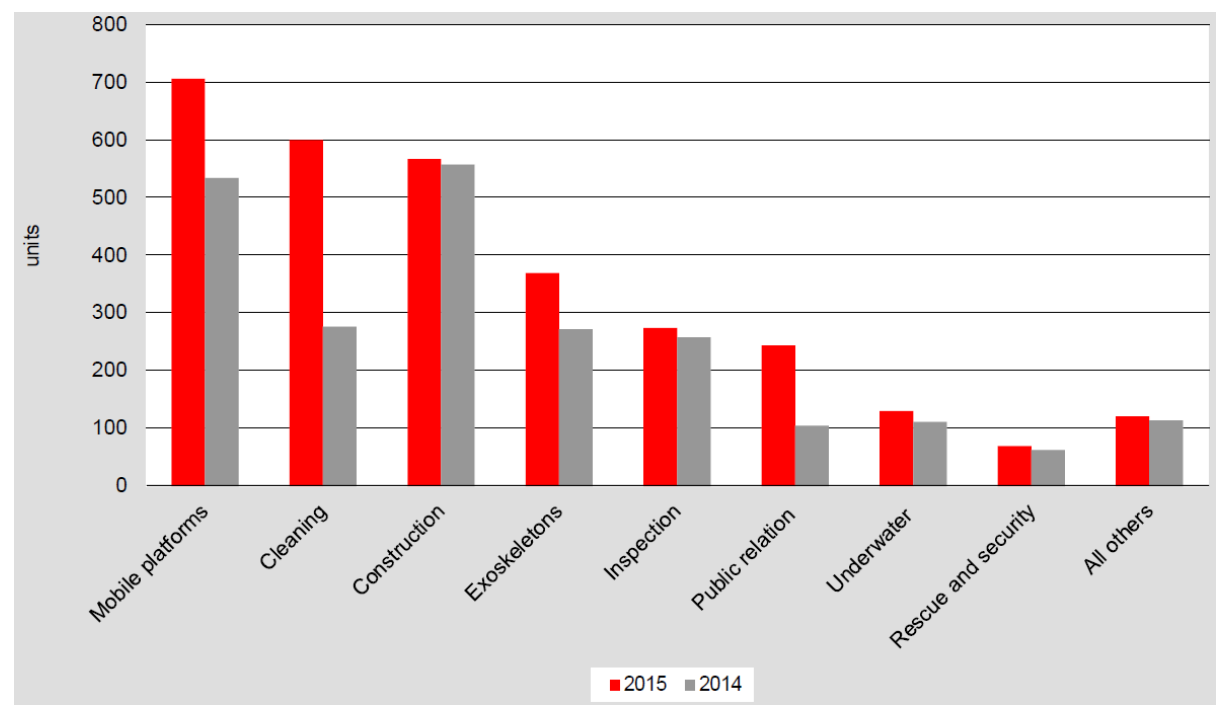

Fig. 1. Service robots for professional use. Sold units 2015 and 2014

and hospitals. Following the definitions, "a certain degree of autonomy" is required for service robots ranging from partial autonomy, including human-robot interaction, to full autonomy, without operational human-robot intervention. The Executive Summary World of Robotics 2017 Service Robots [2, 3], establishes that service robots sales grew in the year 2016 by $24 \%$ (59700 units) compared to the year 2015 (48018 units). The projection for the year 2017 estimates a growth of about 17\% (78700 units), for the years 2018 to 2020 (397000 units) an annual increase is expected between $20 \%$ and $25 \%$.

The International Federation of Robotics (IFR) [4] provides us with a more detailed statistical picture of sales by areas of professional service robotics in units sold during the years 2014 and 2015. As observed in Fig. 1, mobile platforms and robots for cleaning tasks are the two more demanded areas. These figures demonstrate the potential for growth in professional service robotics and a tangible reason for development in this field of robotics.

According to the ISO 8373:2012 standard [1] our proposal about developing a socially-aware robot assistant for serving tasks corresponds to the definition of professional service robots such as in detail restaurants, hotels or any commerce where it is required to move objects from one place to another. Specifically, this proposal is devoted to develop a professional service robot as a delivery assistant. Hence, the main task to be developed by this service robot will be to collaborate with people in the service task by transporting indoors any object (considering the restrictions of the prototype) from one place to another, avoiding obstacles and people on their way.

This service task could seem a simple task to achieve, but the complexity of interacting in social environments is currently one of the most complex problems 
for robots. A number of previous cases can be found in the literature: from the one developed in China [5] with a total failure of the waiter robots with regard to the expectations of the users, to the experience in the Barcelona-based coffeee shop Costa Coffee [6] where successful small demonstrations are working, but it has not been possible to use the proposed robot for prolonged periods.

Our starting hypothesis, based on these past experiences and other similar ones is that the criteria of the user is a fundamental part of the development of this kind of robotic platforms.

The main objective of this work is to define the development of socially-aware robot assistants for serving tasks as delivery in collaboration with a human being from the perspective of social robotics. We defend that this approach will help to overcome past drawbacks in the implementation of service robots in public domains. Moreover, the taxonomy proposed can also be applied to other robotic platforms, contexts and services.

The rest of the article is organized as follows: Section II presents previous cases of the implementation of waiter robots, section III analyzes the social approach in robotics service, finally in section IV presents the first prototype in terms of its minimum hardware and software requirements and approach that we think should be applied in development of this kind of service robot.

\section{Material and Method}

Among the conceptualization of service robots [7] similarly to that exposed previously, Social Robots are distinguished in three different groups:

- Assistive Robots (AR), largely referred to robots which can assist people with physical disabilities through physical interaction.

- Socially Interactive Robots (SIR), describing robots for which social interaction plays a key role.

- Socially Assistive Robots (SAR) as the intersection of assistive robotics (AR) and socially interactive robotics (SIR).

Our revision of the state of the art will be focused on Social and Service Robots evaluated in tasks in restaurants, that is a common area for Professional Service Robots and Socially Interactive Robots.

The following cases can be listed as pioneering attempts to introduce robots in restaurants (see Fig. 2):

- The Hajime robot restaurant in Thailand was open on April 2010 with a robotic waiter-staff of four robots with a total cost of 150,600 euros HAJIME. Two of the robots are endowed with one arm, being in charge of taking the dishes off the kitchen and handing them to their most advanced companions, with two members, mobile body and samurai clothing. They move in the restaurant along a rail. Among the reported problems, there is confusion when removing the finished dishes, serve drinks or collect the bill. 


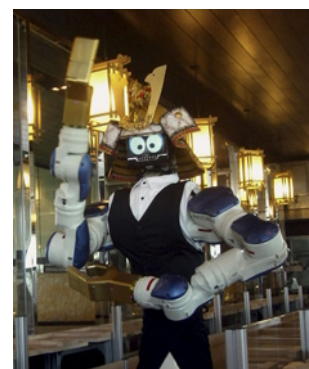

(a) Robot Hajime restaurant.

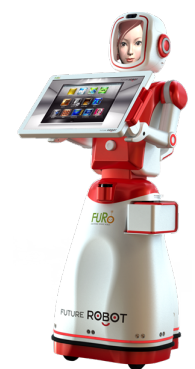

(b) FURO Robot

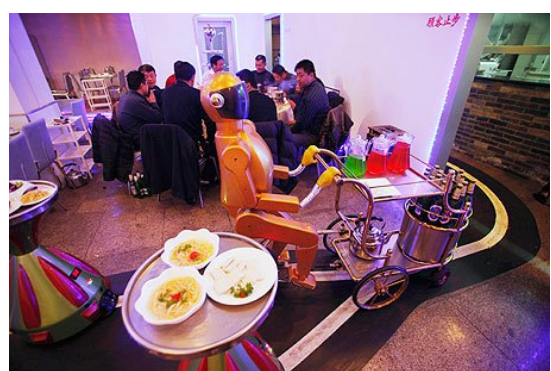

(c) Dalu Hotpot Robot Restaurant.

Fig. 2. Pioneering restaurant robots.

- On June 2010, the FU-RO restaurant robot (Future Robot Co. Ltd) is opened as a practical restaurant robot with hands-on experience [7]. Its service was implemented to make possible interaction between robot and customers through specialized HRI. The company incorporates several robots with different associated tasks such as greeting robot at an entrance, waiting time robot which provides entertainment, order guide robot for guidance, taking order, and payment robot that help customers pay after meals.

- On December 2010, the Dalu Hotpot Robot Restaurant in China [7] had unveiled seven mechanical custom built robot servers with some resemblance to the classic automatons from 1950s science fiction, and others that are basically wheeled tables.

Currently, from these initial experiences, there is only information and availability of the robot in the FU-RO restaurant. In the other two cases, it is unknown whether they were continuing operating.

In [8] the KeJia robot is introduced. This robot plays the role of a bartender by recognizing and grasping the drink when following the order of people and deliver it to a TurtleBot. Several TurtleBots deliver drinks to people as waiters. The human-robot interaction design for service robots being able of safely and effectively interacting with humans is still a challenging problem in robotics. In the KeJia-TurtleBot system, each robot endows some basic behaviors such as navigation or mobile manipulation. These basic behaviors are combined in several ways to yield more complex social behaviors. The Human-Robot Dialogue module provides the interface for communication between users and the robot. The overall system is illustrated in Fig. 3.

According to this experience, in order to optimize the price-performance relationship, the robotic platform TurtleBot 2 will be considered in our proposal for the implementation and testing of the developed solution (see Fig. $3 \mathrm{c}$ ). The mobile base is Kobuki and its cost is $\$ 2.106$.

In [9], a prototype of mobile waiter robot based on Mecanum wheels is introduced. Unlike existing waiter robots, it is able to carry a higher payload. Its development was based on using ROS middleware and implementing SLAM for 


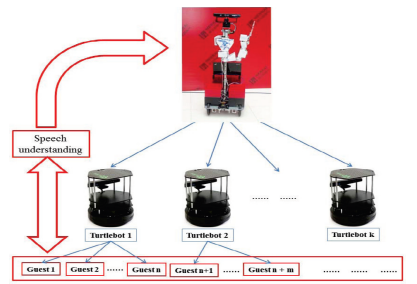

(a) The architecture of the KeJia-Turtelbot system for bartender-waiter application.

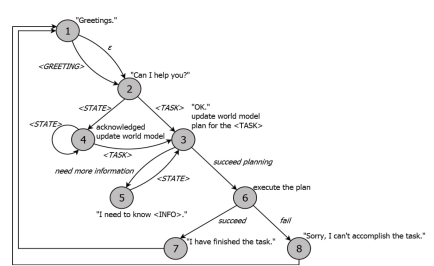

(b) The finite state machine for a simple human-robot dialogue

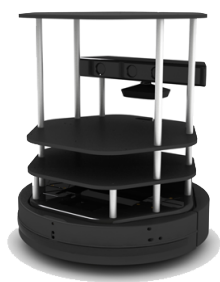

(c) Turtlebot 2

Fig. 3. The KeJia-TurtleBot overall system.

autonomous navigation. Technologically, the robot is robust in its performance however neither robot human interface, nor the social context are considered. Its cost is about $\$ 17.000$. The shape of the prototype can be appreciated in Fig. 4 .
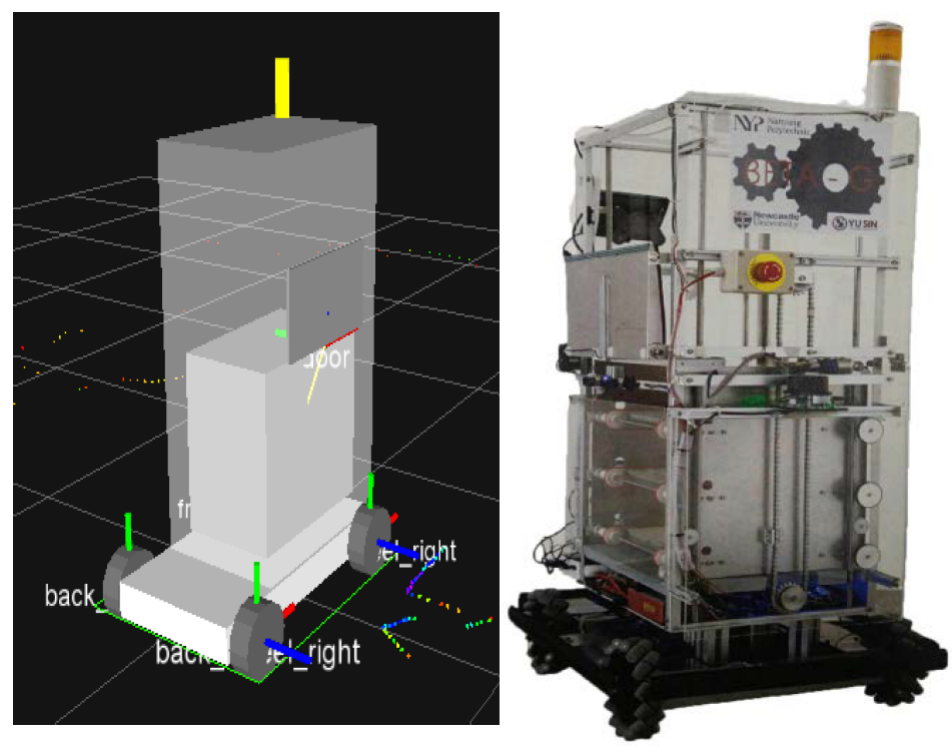

Fig. 4. URDF (left) and actual robot prototype Beta G (right)

The robot Savioke (see Fig. 5 a) was introduced in [10] as a service robot for deliveries and logistics, claiming that they have this robot running in more than 70 hotels. As far as we reviewed the literature, this is the only robot that causes a positive effect considering a social robotics approach. Among its main functions, transportation for sending and receiving items such as towels, toothpaste, 


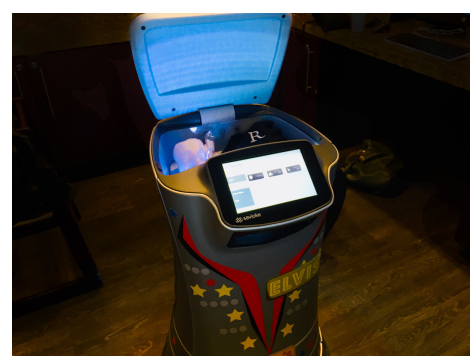

(a) Savioke Robot.

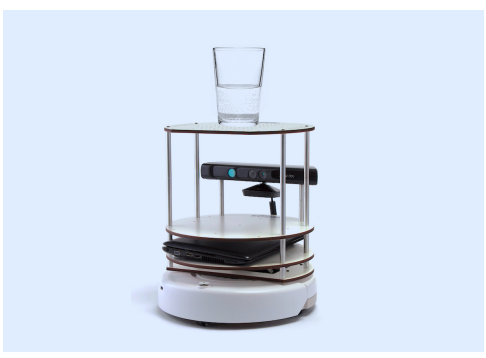

(b) IEEE Spectrum; Robot: TurtleBot; Glass: Jan/Flickr

Fig. 5. Robots from the Kobuki base

newspaper and other room service are well developed, it also has autonomous navigation and the ability to communicate with elevators wirelessly. Nevertheless, the entry and withdrawal of objects must be made by humans.

In [11], an investigation on how we should give orders to a robot is presented. Specifically, they used a TurtleBot remotely commanded to do their movements (see Fig. 5 b) and its voice was generated by a text-to-speech system. This robot is provided with an Artificial Intelligence (AI) system to interact with people. During experimentation the robot played the role of a waiter. The results show that people are overly polite in their order, for example: "Can you Bring Me?" or "If you could X Bring me, that would be great." Instead of direct phrases like" Bring Me X". So the AI system shows that it is still too literal in its interpretation of orders.

\subsection{Focusing on Social Robotics}

A complete review of social robotics is presented in [12]. Due to the extensiveness of the article and relevance of the information presented, we are exposing it graphically in Fig. 11 and only detailed information is evaluated about sections considered relevant to be applied in our proposal. These areas are highlighted with red color to identify the applicable fields to our project according to this taxonomy.

Design Approaches. Talking about design approaches, the Functionally designed approach from [12] will be employed for our proposal. The objective of this approach is to design a robot that outwardly appears to be socially intelligent even if the internal design does not have a basis in science or nature. Hence, design must show the mechanisms, sensations, and traits according to the People's psychology where they understand that a creature is socially intelligent.

Design Issues. We will focus on the Real-time performance of the socially interactive robots operating at human interaction rates. Thus, a robot needs to 


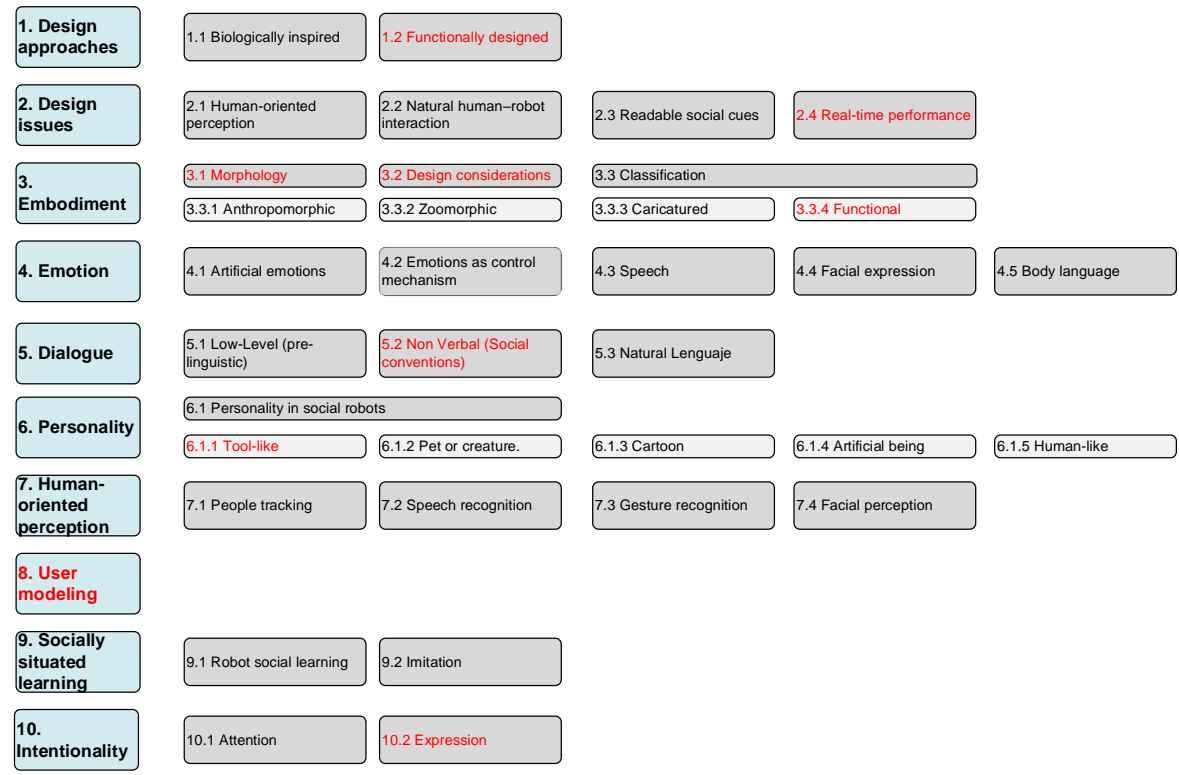

Fig. 6. A proposed taxonomy in Social Robotics.

simultaneously exhibit competent behavior, convey attention and intentionality handle social interaction.

Embodiment. It will be considered from two perspectives: Morphology because the form and structure of a robot is very important considering that it helps to establish social expectations. Design Considerations: since a robot is designed to perform tasks for the human being, then its shape must convey an amount of "productivity" so the user will feel comfortable. Moreover, a robot's design needs to reflect an amount of "robot-ness". This is needed so that the user does not develop detrimentally false expectations of the robot's capabilities.

On the embodiment basis, a robot can be classified as "Functional". The robot's embodiment should first, and foremost, reflect the tasks it must perform. The choice and design of physical features are thus guided purely by operational objectives, especially service robots.

Dialogue. It is an important subject in social robots. The most viable option in our proposal is "Non Verbal (Social conventions)". There exist many non-verbal forms of language, including body positioning, gesturing, and physical action. As most robots have fairly rudimentary capability to recognize and produce speech, non-verbal dialogue is a useful alternative.

At this point it is important to mention the work [13]. The main scope of this work is the idea of expression, which we think about as externalizing hidden information of an agent, in our case a mobile robot. This proposal analyzes the 

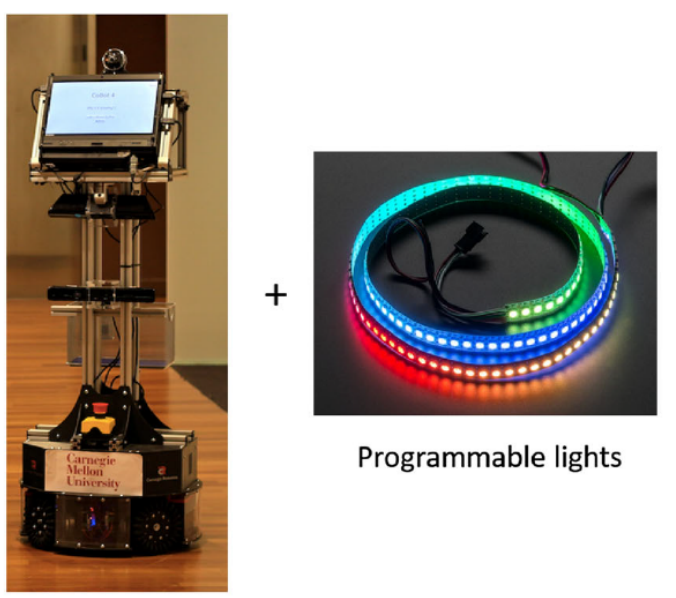

Programmable lights

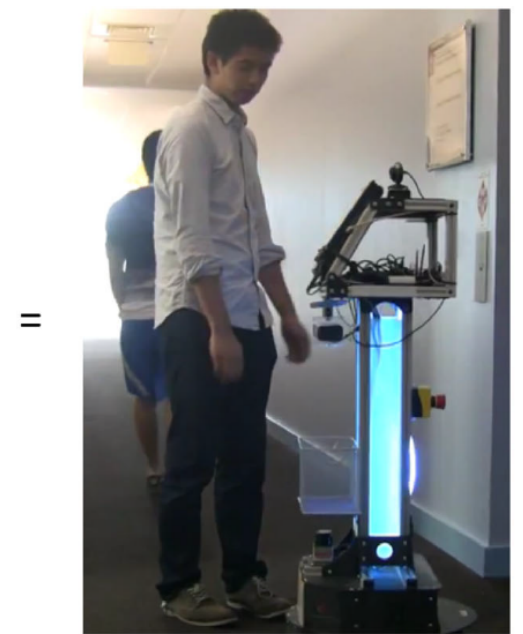

Robot state revealing through expressive lights

Fig. 7. Augmenting a mobile robot's communication capabilities with a new modality: expressive lights.baraka 2017

implementation of LED lights (see Fig. 7) as a means of communication between the mobile robot and humans. Some advantages of this system are its simplicity, cost and ease of communicating at a distance. This is the best option for HRI in our proposal.

Social conventions [12] or norms can also be expressed through non-verbal dialogue. Proxemics, the social use of space, is such one convention. To solve the problems of the proxemics we will use the results tested in [14-16].

Personality. It is organized in social robots into five groups: Tool-like, Pet or creature, Cartoon, Artificial being, Human-like. The "Tool-like" is the one of our interest, that used for robots that operate as smart appliances. Because these robots perform service tasks on command, they exhibit traits usually associated with tools.

User models. They are employed for different purposes. In our proposal user models will be useful for adapting the robot's behavior to suit users with various skills, experience, and knowledge.

Intentionality. It is the last topic to be considered. In detail, "Expression" is understood as the intentionality a robot must exhibit in goal-directed behavior. In our proposal this is considered from its structure and physical aspect for users to understand when seeing the robot which its function and capabilities are.

An important article within the approach of service robotics is [17] the one where they address one of the most important topics for this research pro- 
posal such as the non-use of service robots. This research carried out with 70 autonomous robots for 6 months in the houses of different groups of people, explains the reasons why people stop using them or refuse to use them. Among its most relevant conclusions it enunciates:

- Investigating user's motivations and reasons to refuse or abandon the use of a robot providing important insights for the design and acceptance of robots in our society.

- The challenge for robot designers is to create robots that are enjoyable and easy to use to capture users in the short-term and that are also functionally relevant to keep those users in the longer-term.

This reaffirms the users approach as the main participant in the development of a social robot delivery assistant.

\section{Results}

Based on state of art, we will define the following hardware and software requirements for the developing of the prototype that could be improved based on the user's criteria.

\subsection{Hardware}

The economic side is one of the aspects that will be considered in this research. The cost of the robot must be accessible to the purchasers, to contemplate the return of their investment in areas such as: publicity, the service it lends and the increase of clients due to this implementation.

Considering that price is one of the major constraints for service robot users, the hardware should be the minimum necessary for the robot to provide functionality and not being excessively expensive. The constituent elements of the robot (see Fig. 10) are listed below.

- Robot Platform: Turtlebot 2 Kobuki base.

- Hard Disk: 500 GB HDD

- Controller: Intel NUC I5

- 3D Sensor: Orbbec Astra

- 2D Sensor: RPLIDAR A3 Lasser Scanner

- Additional battery: 4S2P (4400 mAh)

- Screen and Touch: GeChic 13.3" visualización led-lit Monitor (GeChic 1303i)

For the listed hardware the average cost is $\$ 3,000$ which is obviously still lower compared to commercial platforms such as iROBOT ROOMBA. However, it is within the feasible costs of a prototype. The selection of the Turtlebot 2 with its Kobuki platform is a low-cost platform that nevertheless meets the minimum characteristics that are necessary to implement our prototype for indoor testing. Among the most important features we mention: 


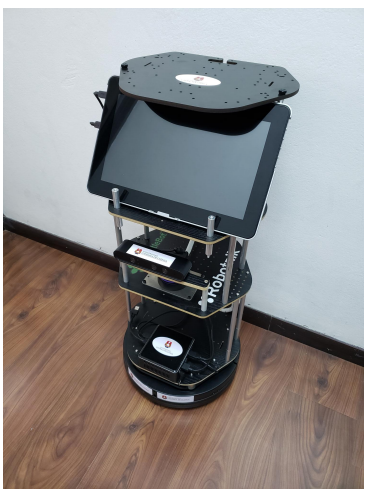

(a) Robot Left.

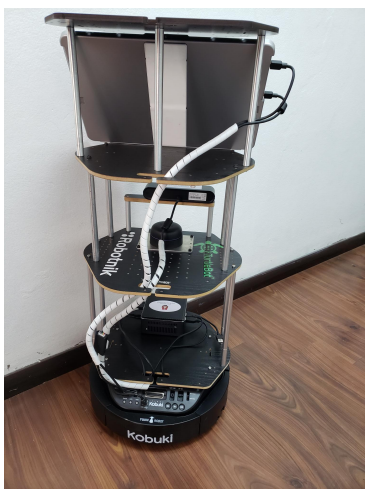

(b) Robot Back.

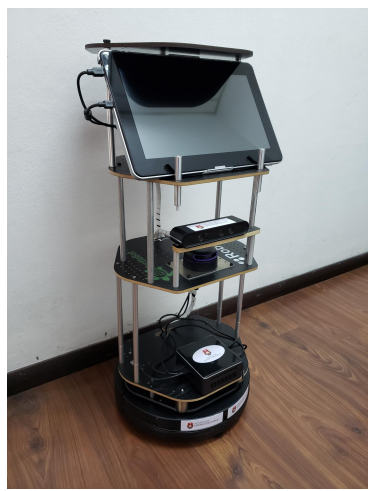

(c) Robot Right.

Fig. 8. First Prototype: basic structure.

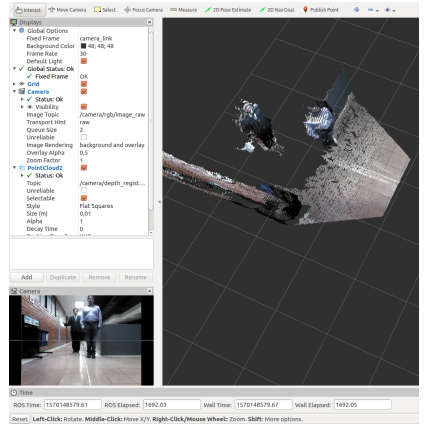

(a) 3D Camera.

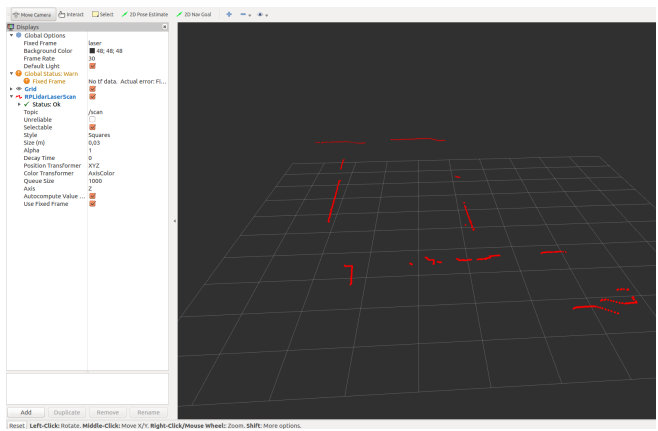

(b) LIDAR.

Fig. 9. TEST.

- Maximum linear speed: $70 \mathrm{~cm} / \mathrm{s}$.

- Maximum angular speed: $180 \mathrm{deg} / \mathrm{s}$.

- Load capacity: $5 \mathrm{~kg}$ (hard floor), $4 \mathrm{~kg}$ (carpet).

- Vertical slopes: detects slopes greater than $5 \mathrm{~cm}$ in height.

- Maximum unevenness: overcomes unevenness up to $12 \mathrm{~mm}$

- Operating time: 7 hours (large battery)

- Charging time: 2.6 hours (large battery)

Orbbec Astra is a 3D camera compatible with OpenNI which is a package used in ROS to manage this type of cameras to help during the time of implementation and tuning with SLAM algorithms. The detection ranges of the camera go from 0.4 to 8 meters. In the tests performed with people, our results were as follows. While standing the Camera position at $49 \mathrm{~cm}$ from the floor, the minimum distance was $0.12 \mathrm{~m}$, the maximum was $5 \mathrm{~m}$ and the opening angle was 68 degrees. Probably the obstacle detection with the 3D camera is not enough 


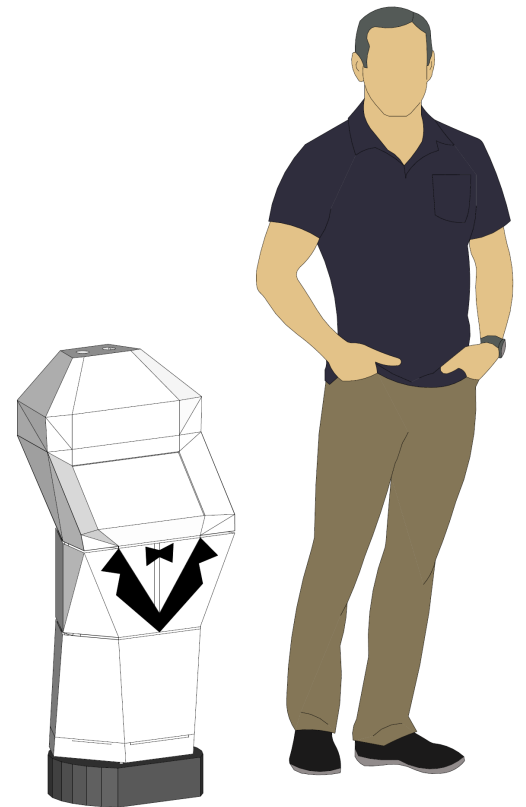

(a) Human Comparison.

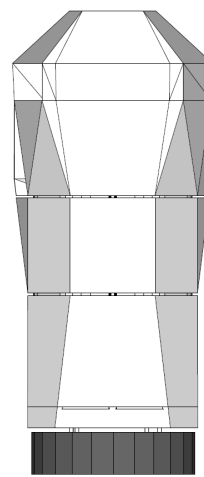

(b) Back.

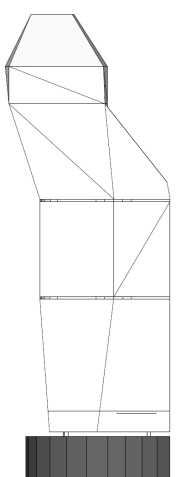

(c) Right.

Fig. 10. First Prototype: Body Proposal.

so we considered to include a ROS compatible Lidar sensor $2 \mathrm{D}$ that uses the Rplidar package. The main features of this LIDAR sensor are:

- Scanning Angle: 360 deg.

- Distance range: 10-25 m.

- Scan Speed: 10-20 Hz.

- Angular resolution: $0.3375 \mathrm{deg}, 0.54 \mathrm{deg}$.

To perform the following tests with people, the LIDAR was placed $43 \mathrm{~cm}$ from the floor. The results allowed us to show that people wearing black clothes were detected at a maximum range of $6 \mathrm{~m}$. The maximum range of detection of people wearing other colors than black was $25 \mathrm{~m}$. In both cases the minimum detection was $0.12 \mathrm{~m}$. The LIDAR was placed $43 \mathrm{~cm}$ from the floor.

The design of the Robot Body must consider the taxonomy shown in Fig. 11. In detail 1.2 Functionally Designed, 2.4 Morphology, 3.2 Design Considerations, 3.3.4 Functional, 6.1.1 Tool-like, 10.2 Expression. For all the reasons above, the design illustrated in figure 9 does not contemplate elements that generate false expectations about the robot. This implies that as it does not have arms, ears or mouth it is not able to manipulate objects, listen to orders and respond verbally. In this first stage it has only the capacity to move objects from point A (A4 folders, coffee cups and others of the same size) to Point B (in the compartment of its upper part on the screen.) On the screen the robot has two eyes that allows 


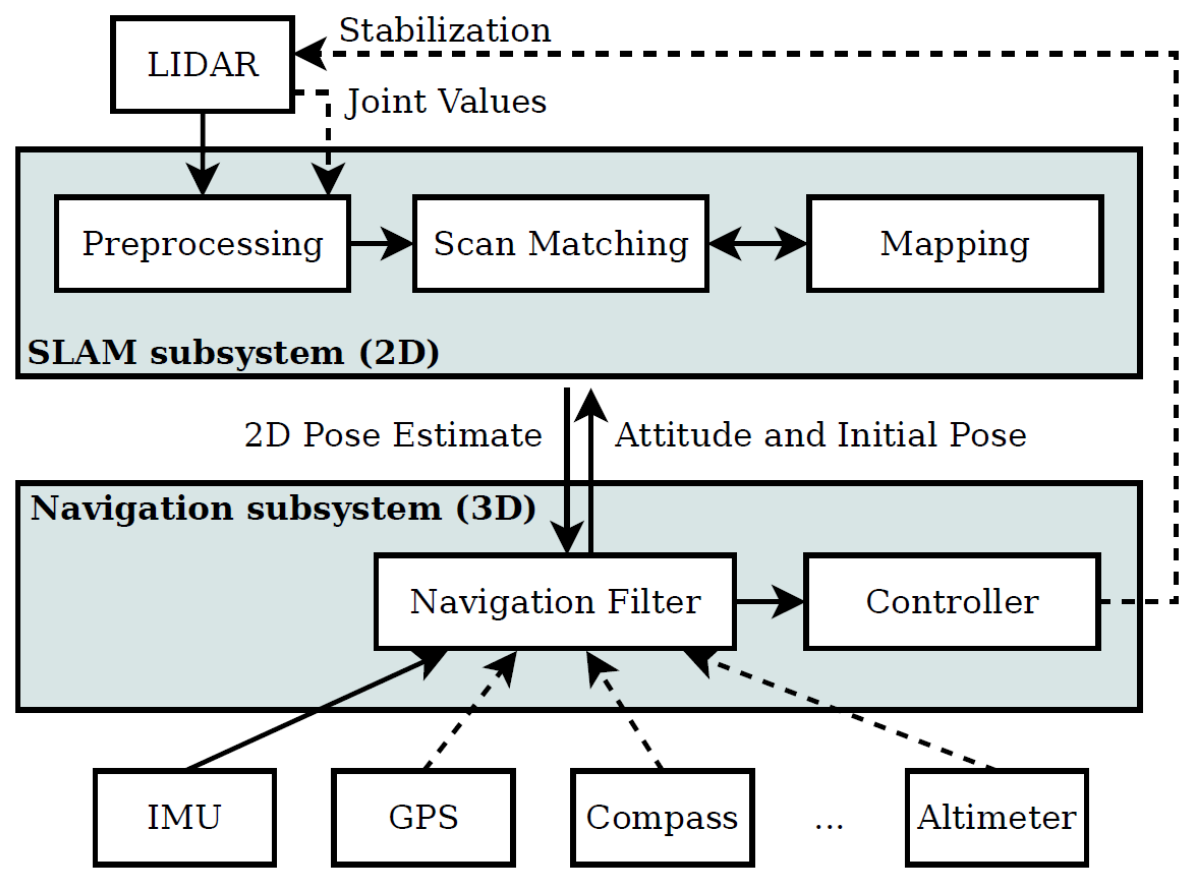

Fig. 11. Mapping and Navigation System. [18]

it to avoid obstacles and fulfill the assigned trajectory. These fulfill the function of communicating with the user and the ability to perceive their environment although this function is performed by the 3D camera and the LIDAR.

Finally as controller we select an Intel NUC I5 is a $4 \mathrm{x} 4$-inch mini PC, with 8 gigabytes of ram and we include as interface for the user a portable Touch Screen GeChic of 13,3 inches.

\subsection{Software}

While working on robot development, open source platforms are the most popular choice. The problem with these platforms is that they are constantly being tested, improved and generally have compatibility problems with the newer peripherals. Considering all the constraints exposed the following development tools were selected:

- Operating System: Ubuntu 16.04 LTS.

- Framework: ROS Kinetic Kame.

- Additional Packages: OpenNI, Rplidar, Hector Slam, Gmapping.

In the software considerations Ubuntu version with long term support 16.04 was used and not the 18.04 because there are not several ROS packages compatible and necessary for this proposal. ROS Kinetic is the latest version of ROS 
with supported packages (OpenNI, Rplidar, Hector Slam, Gmapping) for minimum implementation on the TurtleBot 2. The main task for this robot platform is the transport of objects from point A to point B. To perform this task the Robot needs to have a map of the place in which it will move and be able to avoid obstacles whether fixed or mobile as in the case of people. On the basis of the articles [19-22] SLAM (Simultaneous Localization and Mapping) will be applied to carry out our task. This is due to the fact that with SLAM we could make an initial map of the space where the robot will make its displacements and additionally provides information about fixed and mobile obstacles.

The article [18] presents the option that best suits our proposal.This is because it presents a package developed for ROS that integrates a $2 \mathrm{D}$ sensor (LIDAR) and another 3D for the generation of maps using SLAM see Fig. 11.The most interesting part of [18] is the integration of the 2D and 3D sensor for SLAM. The 3D estimation applies Kalman Filter, the results of both sensors are not synchronized, the $3 \mathrm{D}$ estimation is superimposed on the $2 \mathrm{D}$ results.

\section{Conclusion}

The technological considerations are always the most important for the design of a robot in the work of the engineers. However, this work tries to show that the social considerations are essential to achieve a robot that is accepted and used by the people that are not engineers.

An explicit contribution of this analysis is the taxonomy in graphic form of the social robots. In a specific way, the aspects relevant to the proposal for the development of a robot assistant are analyzed.

Results section proposes a minimum platform for development considering the engineering aspects such as hardware and software.

Articles such as [17] reaffirm that user-focused design is what is needed to overcome the resistance of people to the use of service robots.It is still an outstanding task to establish metrics that allow measuring the performance of a service robot in a social environment.

Within the expected contributions it is proposed to develop a robust robotic platform, which satisfies the expectations of the users. If these expectations are not satisfied the robot will not be used, therefore the objective is not fulfilled.

Another contribution that is expected to be obtained in the research is to obtain an experience in service robotics in social environments, for periods greater than the aforementioned cases. 


\section{References}

1. International Federation of Robotics. Service Robots - Definition and Classification WR 2016. pages 9-12, 2012.

2. International Federation of Robotics. Executive Summary World Robotics 2017 Service Robots. Executive Summary World Robotics 2017 Service Robots, pages 12-19, 2017.

3. Mingming Guo, Hua Zhang, Guanzhi Zhang, and Jianwen Huo. La utopía posible: Tecnología futurista y calidad de vida. Integración ES comercio, (42):216-230, 2017.

4. International Federation of Robotics. Executive Summary World Robotics 2016 Service Robots. Executive Summary World Robotics 2016 Service Robots, pages $1-5,2016$.

5. Chuck Thompson, Elaine Yu, CNN. New order? China restaurant debuts robot waiters. http://edition.cnn.com/travel/article/china-robot-waiters/index. html. Accessed: 2016-04-19.

6. RestauracionNews. Costa coffee presenta el primer robot barista del mundo. http://www.restauracionnews.com/ costa-coffee-presenta-primer-robot-barista-del-mundo/. Accessed: 2017-07-01.

7. Sakari Pieskä, Mika Luimula, Juhana Jauhiainen, and V Spitz. Social service robots in public and private environments. Recent Researches in Circuits, Systems, Multimedia and Automatic Control, pages 190-196, 2012.

8. Qiang Lu, Guanghui Lu, Aijun Bai, Dongxiang Zhang, and Xiaoping Chen. An intelligent service system with multiple robots. In Robot Competition of International Joint Conference on Artificial Intelligence (IJCAI 2013), 2013.

9. A Cheong, MWS Lau, E Foo, J Hedley, and Ju Wen Bo. Development of a robotic waiter system. IFAC-PapersOnLine, 49(21):681-686, 2016.

10. Tekla S. Perry. Ces 2018: Delivery robots are full-time employees at a las vegas hotel. https://spectrum.ieee.org/view-from-the-valley/robotics/industrial-robots/ ces-2018-delivery-robots-are-fulltime-employees-at-a-las/-vegas-hotel? Accessed: 2018-01-12.

11. Evan Ackerman, Ieee International Conference, and Human Robot Interaction. How Not to Order Water from a Robot Waiter. pages 1-5, 2018.

12. Terrence Fong, Illah Nourbakhsh, and Kerstin Dautenhahn. A survey of socially interactive robots. Robotics and autonomous systems, 42(3):143-166, 2003.

13. Kim Baraka and Manuela M Veloso. Mobile service robot state revealing through expressive lights: Formalism, design, and evaluation. International Journal of Social Robotics, pages 1-28, 2017.

14. Harmish Khambhaita and Rachid Alami. Assessing the social criteria for humanrobot collaborative navigation: A comparison of human-aware navigation planners. In Proc. IEEE International Symposium on Robot and Human Interactive Communication (RO-MAN), page 6p, 2017.

15. Thibault Kruse, Amit Kumar Pandey, Rachid Alami, and Alexandra Kirsch. Human-aware robot navigation: A survey. Robotics and Autonomous Systems, 61(12):1726-1743, 2013.

16. Jorge Rios-Martinez, Anne Spalanzani, and Christian Laugier. From proxemics theory to socially-aware navigation: A survey. International Journal of Social Robotics, 7(2):137-153, 2015.

17. Maartje de Graaf, Somaya Ben Allouch, and Jan van Dijk. Why do they refuse to use my robot?: Reasons for non-use derived from a long-term home study. In Proceedings of the 2017 ACM/IEEE International Conference on Human-Robot Interaction, pages 224-233. ACM, 2017. 
18. S. Kohlbrecher, J. Meyer, O. von Stryk, and U. Klingauf. A flexible and scalable slam system with full $3 \mathrm{~d}$ motion estimation. In Proc. IEEE International Symposium on Safety, Security and Rescue Robotics (SSRR). IEEE, November 2011.

19. Hesham Ibrahim Mohamed Ahmed Omara and Khairul Salleh Mohamed Sahari. Indoor mapping using kinect and ros. In 2015 International Symposium on Agents, Multi-Agent Systems and Robotics (ISAMSR), pages 110-116. IEEE, 2015.

20. Arbnor Pajaziti. Slam-map building and navigation via ros. International Journal of Intelligent Systems and Applications in Engineering, 2(4):71-75, 2014.

21. Monserrat Rojas-Fernández, Dante Mújica-Vargas, Manuel Matuz-Cruz, and Diana López-Borreguero. Performance comparison of $2 \mathrm{~d}$ slam techniques available in ros using a differential drive robot. In 2018 International Conference on Electronics, Communications and Computers (CONIELECOMP), pages 50-58. IEEE, 2018.

22. Hamza Aagela, Maha Al-Nesf, and Violeta Holmes. An asus_xtion_probased indoor mapping using a raspberry pi with turtlebot robot turtlebot robot. In 2017 23rd International Conference on Automation and Computing (ICAC), pages 1-5. IEEE, 2017. 